OPEN ACCESS

Edited by:

Michael Gänzle

University of Alberta, Canada

Reviewed by:

Christopher Doona,

US Army - Natick Soldier RDEC, USA

Sergio I. Martinez-Monteagudo,

The Ohio State University, USA

*Correspondence:

Peng Dong,

China Agricultural University, Qinghua East Road 17, Beijing 100083, China

dongpeng12@hotmail.com

Specialty section: This article was submitted to

Food Microbiology,

a section of the journal

Frontiers in Microbiology

Received: 07 April 2015

Accepted: 29 June 2015

Published: 14 July 2015

Citation:

Dong P, Georget ES, Aganovic K,

Heinz $V$ and Mathys A (2015) Ultra

high pressure homogenization (UHPH)

inactivation of Bacillus

amyloliquefaciens spores in

phosphate buffered saline (PBS) and

milk. Front. Microbiol. 6:712.

doi: 10.3389/fmicb.2015.00712

\section{Ultra high pressure homogenization (UHPH) inactivation of Bacillus amyloliquefaciens spores in phosphate buffered saline (PBS) and milk}

\author{
Peng Dong 1, 2,3,4*, Erika S. Georget ${ }^{4,5}$, Kemal Aganovic ${ }^{4}$, Volker Heinz ${ }^{4}$ and \\ Alexander Mathys ${ }^{4}$
}

${ }^{1}$ College of Food Science and Nutritional Engineering, China Agricultural University, Beijing, China, ${ }^{2}$ National Engineering Research Center for Fruits and Vegetables Processing, Beijing, China, ${ }^{3}$ Key Laboratory of Fruits and Vegetables Processing, Ministry of Agriculture, Beijing, China, ${ }^{4}$ German Institute of Food Technologies, Quakenbrück, Germany, ${ }^{5}$ Institute of Food Chemistry, Leibniz Universität Hannover, Hannover, Germany

Ultra high pressure homogenization (UHPH) opens up new areas for dynamic high pressure assisted thermal sterilization of liquids. Bacillus amyloliquefaciens spores are resistant to high isostatic pressure and temperature and were suggested as potential surrogate for high pressure thermal sterilization validation. B. amyloliquefaciens spores suspended in PBS buffer (0.01 M, pH 7.0), low fat milk (1.5\%, pH 6.7), and whole milk $(3.5 \%, \mathrm{pH} 6.7)$ at initial concentration of $\sim 10^{6} \mathrm{CFU} / \mathrm{mL}$ were subjected to UHPH treatments at 200,300 , and $350 \mathrm{MPa}$ with an inlet temperature at $\sim 80^{\circ} \mathrm{C}$. Thermal inactivation kinetics of $B$. amyloliquefaciens spores in PBS and milk were assessed with thin wall glass capillaries and modeled using first-order and Weibull models. The residence time during UHPH treatments was estimated to determine the contribution of temperature to spore inactivation by UHPH. No sublethal injury was detected after UHPH treatments using sodium chloride as selective component in the nutrient agar medium. The inactivation profiles of spores in PBS buffer and milk were compared and fat provided no clear protective effect for spores against treatments. Treatment at $200 \mathrm{MPa}$ with valve temperatures lower than $125^{\circ} \mathrm{C}$ caused no reduction of spores. A reduction of 3.5 $\log _{10} \mathrm{CFU} / \mathrm{mL}$ of $B$. amyloliquefaciens spores was achieved by treatment at $350 \mathrm{MPa}$ with a valve temperature higher than $150^{\circ} \mathrm{C}$. The modeled thermal inactivation and observed inactivation during UHPH treatments suggest that temperature could be the main lethal effect driving inactivation.

Keywords: ultra high pressure homogenization, bacterial spore, Bacillus amyloliquefaciens, inactivation, milk, fat content 


\section{Introduction}

Bacterial spores pose a major hazard in food safety because of their high resistance to most hurdles. Thermal sterilization is an effective method to inactivate bacterial spores, however, it has negative effects on sensorial and nutritional qualities of foods (Reineke et al., 2013; Georget et al., 2014b). Hence, alternative preservation technologies have been investigated to inactivate bacterial spores while retaining sensorial and nutritional properties, such as high isostatic pressure (Mathys et al., 2007a; Reineke et al., 2011a, 2013; Georget et al., 2015), pulsed electric fields (Siemer et al., 2014a,b; Toepfl et al., 2014), ultra-violet light (Baysal et al., 2013; Gayán et al., 2013), and ultrahigh pressure homogenization (UHPH) (Georget et al., 2014a,b). Conventional homogenizers with pressures up to $50 \mathrm{MPa}$ have been used in beverages, pharmaceutical and cosmetic industries to reduce particle size and produce stable emulsions but are not sufficient to induce bacterial spore inactivation. It is suspected that this is due to the low homogenization pressure and the resulting low valve temperatures. The recent enhancement of homogenization pressure up to $400 \mathrm{MPa}$, within which pressure range $>200 \mathrm{MPa}$ can be regarded as UHPH, has opened up new areas for dynamic pressure assisted thermal sterilization of liquids (Diels and Michiels, 2006; Georget et al., 2014b). UHPH is a continuous process that has potential application in the processing of liquid foods including juices, vegetable milk, and dairy products. The inactivation of microorganisms is achieved by UHPH and thus the shelf-life can be extended. The products are of improved sensorial and nutritional characteristics, among others, because of the increased emulsion stability and very short residence time at high pressure and/or high temperature (Zamora and Guamis, 2015).

Inactivation of different bacterial spores in model and food systems by UHPH has been reviewed and the overview highlights a varying success (Georget et al., 2014b). The naturally present spores in milk with $3.5 \%$ fat decreased from 1.7 to $0.6 \log _{10} \mathrm{CFU} / \mathrm{mL}$ after treatment at $300 \mathrm{MPa}$ with valve temperature at $103^{\circ} \mathrm{C}$ (Pereda et al., 2007). For Geobacillus stearothermophilus ATCC7953 and Clostridium sporogenes PA3679 spores in skim milk, a UHPH treatment with 16 passes at $300 \mathrm{MPa}$ with an inlet temperature at $45^{\circ} \mathrm{C}$ caused a reduction of only $0.67 \log _{10} \mathrm{CFU} / \mathrm{mL}$ (Pinho et al., 2011). However, several successful investigations were also reported. It was found that up to $5 \log _{10} \mathrm{CFU} / \mathrm{mL}$ Bacillus subtilis PS832 and $2 \log _{10} \mathrm{CFU} / \mathrm{mL}$ G. stearothermophilus ATCC7953 spores suspended in phosphate buffered saline (PBS buffer) could be inactivated by single pass UHPH treatments applying pressures higher than $300 \mathrm{MPa}$ and valve temperatures higher than $145^{\circ} \mathrm{C}$ with estimated holding times below $0.5 \mathrm{~s}$. It was concluded that the very high valve temperatures could be a dominant parameter leading to bacterial spores' inactivation (Georget et al., 2014a). In another investigation, commercial whole milk with $3.6 \%$ fat was inoculated with Bacillus cereus, Bacillus licheniformis, Bacillus sporothermodurans, Bacillus coagulans, G. stearothermophilus, and B. subtilis spores. UHPH treatments at $300 \mathrm{MPa}$ with inlet temperature at 75 and $85^{\circ} \mathrm{C}$ were capable of a reduction of $5 \log _{10} \mathrm{CFU} / \mathrm{mL}$ (Amador Espejo et al.,
2014). These recent investigations suggest a high potential of UHPH for single stage dynamic pressure assisted thermal spore inactivation.

Most of previous studies were conducted in buffers or food systems with low inlet temperature, and valve temperatures were not always monitored, which made it difficult to compare the reported results. While strong inactivation can be observed with higher homogenization pressures and inlet (and consequently valve) temperatures, the contribution of individual parameters was not clear and the influence of temperature on the inactivation should also be assessed. Furthermore, it should be noted that results of studies in model systems cannot be directly extrapolated to real food systems. The food constitutes could have an influence on the inactivation of microorganisms by UHPH as it was already shown for high isostatic pressure high temperature microbial inactivation (Georget et al., 2015). Some studies showed that milk fat could protect Listeria monocytogenes and endogenous flora against UHPH treatments (Kheadr et al., 2002; Vachon et al., 2002). Contrasting results were also reported in Escherichia coli suspended in buffers and milk (Diels et al., 2005; Briñez et al., 2006). Little information exists on the influence of milk fat on the inactivation of spores during UHPH treatments, and few comparative studies were found on the inactivation of spores by UHPH in model and food systems. While the investigations on vegetative microorganisms showed that no sublethal injury was observed after UHPH treatments (Wuytack et al., 2002; Briñez et al., 2007; Donsì et al., 2009; RoigSagués et al., 2009), there is a lack of information on the potential occurrence of such sublethal injury for spores following UHPH treatments.

When considering the efficacy of UHPH sterilization process, two important stress factors are underlined: pressure and temperature. It is thus expected that a good challenge strain should have a strong thermal resistance and potentially strong pressure resistance. Limited work was conducted on strains such as G. stearothermophilus and C. sporogenes spores (Pinho et al., 2011; Georget et al., 2014a) and showed that high thermal resistance is an essential attribute of potential surrogate for UHPH spore inactivation. In the present study, Bacillus amyloliquefaciens spores were used due to their resistance to high pressure thermal sterilization (Margosch et al., 2006; Sevenich et al., 2013) thus also potentially making them a challenge strain of interest. Spores suspended in PBS buffer (0.01 M, pH 7.0), low fat milk ( $\mathrm{pH} 6.7)$, and whole milk $(\mathrm{pH} 6.7)$, were treated by a Stansted model FPG11300 UHPH unit. Following this, the UHPH inactivation profiles of spores suspended in PBS buffer and milk were compared. The influence of the fat content on the inactivation of spores was investigated. The thermal resistance of $B$. amyloliquefaciens spores was also assessed by modeling approaches to estimate the contribution of temperature to spore inactivation by UHPH.

\section{Materials and Methods}

\section{B. amyloliquefaciens Spores Preparation}

The bacterial strain used in this study was Bacillus amyloliquefaciens FAD82 (Technische Mikrobilogie 
Weihenstephan, TMW 2.479), courtesy of Professor Michael Gänzle, University of Alberta, Canada. B. amyloliquefaciens spores were obtained following a method described elsewhere (Sevenich et al., 2013). A single colony of B. amyloliquefaciens grown on nutrient agar overnight was inoculated in $10 \mathrm{~mL}$ of nutrient broth. The suspension was incubated at $37^{\circ} \mathrm{C}$ and $250 \mathrm{rpm}$ in a shaking incubator until the required optical density measured at a wavelength of $600 \mathrm{~nm}\left(\mathrm{OD}_{600}=1.6-\right.$ $1.8 /$ control) was reached (controlled with a cell density meter Ultrospec 10, Amersham Biosciences GmbH, Germany). Subsequently, a volume of $200 \mu \mathrm{L}$ of the culture was spread onto $2 \times$ SG medium agar plates without antibiotics. After incubation at $37^{\circ} \mathrm{C}$ for $48 \mathrm{~h}$, sporulation was monitored with a phase contrast microscope daily. When the percentage of sporulation reached more than $95 \%$, the plates were stored at room temperature until the remaining vegetative cells were dried out. The spores were harvested using $4^{\circ} \mathrm{C}$ sterile distilled water and cleaned by repeated centrifugation (four-fold at $4800 \mathrm{~g}$ ) until the supernatant was clean. The spore suspension was additionally treated twice with sonication for $1 \mathrm{~min}(35 \mathrm{kHz}-$ $160 \mathrm{~W}_{\mathrm{eff}}$ ) (Bandelin Sonorex RK $510 \mathrm{H}$, Berlin, Germany) during the cleaning stage. The cleaned spore suspension at the concentration of $\sim 10^{8} \mathrm{CFU} / \mathrm{mL}$ contained $>95 \%$ phase bright spores without agglomerates as was verified by phase contrast microscopy and particle size distribution analysis (Mastersizer 2000, Malvern Instruments Ltd, Malvern, UK) (Table 1). Bacterial spores were stored in the dark at $4^{\circ} \mathrm{C}$ until use.

B. amyloliquefaciens spores were suspended in $0.01 \mathrm{M}$ PBS buffer $\left(137 \mathrm{mM} \mathrm{NaCl}, 2.7 \mathrm{mM} \mathrm{KCl}, 10 \mathrm{mM} \mathrm{Na} 2 \mathrm{HPO}_{4}\right.$ and $1.8 \mathrm{mM} \mathrm{KH} \mathrm{PO}_{4}$, pH 7.0) (Burston et al., 2008), low fat milk (1.5\% fat, Gutesland, Netto Marken-Discount AG \& Co. KG, Germany), and whole milk (3.5\% fat, Gutesland, Netto MarkenDiscount AG \& Co. KG, Germany) at initial concentration of $\sim 10^{6} \mathrm{CFU} / \mathrm{mL}$. PBS buffer was selected because of its high stability at high temperatures and avoided potential $p K a$ variation at high temperatures as was reported in previous work (Reineke et al., 2011b).

TABLE 1 | Particle size distribution analysis of $B$. amyloliquefaciens spores suspension (result transform type: volume).

\begin{tabular}{lc}
\hline Parameters & Values \\
\hline Concentration & $0.0018 \%$ Vol \\
Obscuration & $6.38 \%$ \\
Weighted residual & $5.221 \%$ \\
Specific surface area & $6.35 \mathrm{~m}^{2} / \mathrm{g}$ \\
Uniformity & 0.335 \\
Span & 0.978 \\
Particle density & 1.000 \\
Mode & 0.989 \\
Surface weighted mean D [3,2] & $0.946 \mu \mathrm{m}$ \\
Volume weighted mean D [4,3] & $1.130 \mu \mathrm{m}$ \\
$d(0.1)$ & $0.670 \mu \mathrm{m}$ \\
$d(0.5)$ & $1.023 \mu \mathrm{m}$ \\
$d(0.9)$ & $1.670 \mu \mathrm{m}$
\end{tabular}

\section{UHPH Treatment Conditions}

The UHPH treatments were performed in a pilot-scale unit (model FPG11300, Stansted Fluid Power Ltd, Harlow, UK), and the scheme is shown in Figure 1. This device comprised two high pressure valves, the first of which is a ceramic valve able to support $400 \mathrm{MPa}$ and the second of which maintains a slight back pressure of $\sim 10 \mathrm{MPa}$. The spore suspension was preheated to $\sim 80^{\circ} \mathrm{C}$ by recirculation through a tubular heat exchanger (DIL e.V., Quakenbrueck, Germany) and then processed through a plate heat exchanger (DIL e.V., Quakenbrueck, Germany) and the UHPH unit. The selection of the pre-heating temperature was based on previous work by the authors showing the absence of strong inactivation by UHPH for lower inlet temperatures at 37 and $55^{\circ} \mathrm{C}$ (Georget et al., 2014a). After the first valve, the temperature of the processing medium was decreased below $50^{\circ} \mathrm{C}$ within $<1 \mathrm{~s}$ using glycol water at $-10^{\circ} \mathrm{C}$ as the cooling agent. Samples were collected into sterile tubes and put in an ice bath immediately for subsequent plate counts. For UHPH conditions, the target inlet temperature was $80^{\circ} \mathrm{C}$, and the pressures were 200,300 , and $350 \mathrm{MPa}$. The pressure fluctuation was no more than $10 \mathrm{MPa}$, except two trials for whole milk with treatments at $350 \mathrm{MPa}$, and the measured pressures were 366 and $370 \mathrm{MPa}$. The valve temperature was monitored, which was related to homogenization pressure and inlet temperature $\left(\sim 20^{\circ} \mathrm{C}\right.$ of temperature increase per $100 \mathrm{MPa}$ of pressure applied) and was used to compare to thermal inactivation kinetics based on an estimated residence time at valve temperature before cooling. The estimated residence times in different unit segments were derived from the measured flow rate under UHPH conditions $(95.6 \pm$ $4.2 \mathrm{~L} / \mathrm{h}$ ) and the equipment dimensions. All treatment conditions were repeated in three independent trials with the same initial spore batch.

\section{Thermal Inactivation of $B$. amyloliquefaciens Spores}

To enable quasi isothermal conditions, thin wall glass capillaries were used to measure thermal inactivation kinetics of spores (Mathys et al., 2007b; Mathys, 2008) using the same spore batch as for the UHPH trials. Thin wall glass capillaries with an inner diameter of $1.0 \mathrm{~mm}$, an outer diameter of $1.3 \mathrm{~mm}$, and a length of $300 \mathrm{~mm}$ (Kleinfeld Labortechnik $\mathrm{GmbH}$, Gehrden, Germany) were filled with a spore suspension volume of $100 \mu \mathrm{L}$. The capillaries were thermally treated in a thermostat (HaakeDC50, Karlsruhe, Germany) with silicon oil SIL180 (Thermo Fisher Scientific, Karlsruhe, Germany) as heating medium with temperatures ranging from 105 to $121^{\circ} \mathrm{C}$ and holding time between 15 and $7200 \mathrm{~s}$ depending on the temperature. After the defined holding time, the samples were rapidly cooled in an ice bath to avoid further inactivation. All thermal inactivations were repeated three times using the same spore batch.

\section{Microbiological Analysis}

Following thermal and UHPH treatments, samples were plated on nutrient agar (peptone $5.0 \mathrm{~g}$, meat extract $3.0 \mathrm{~g}$, agar $15.0 \mathrm{~g}$, BD, Le Pont de Claix, France; distilled water 1000.0 mL, pH 7.0) in triplicate and incubated at $37^{\circ} \mathrm{C}$ for $48 \mathrm{~h}$. The sublethal injury 


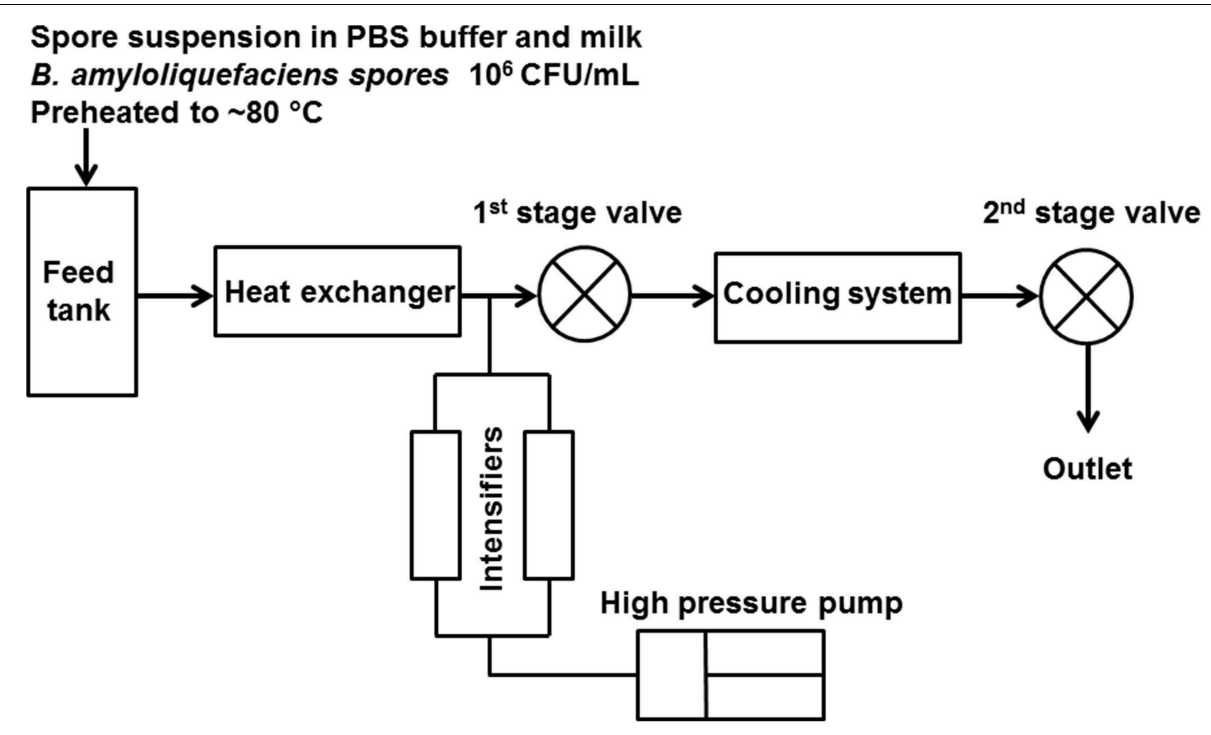

FIGURE 1 | Scheme of the UHPH unit setup (UHPH unit model FPG11300, Stansted Fluid Power Ltd, Harlow, UK).

of spores was estimated by comparing colonies on nutrient agar alone and nutrient agar supplemented with $6 \%$ sodium chloride as a selective medium $(\mathrm{NaCl})$. Preliminary experiments with different $\mathrm{NaCl}$ concentrations ranging from 3 to $12 \%$ showed that the maximum concentration that did not affect the growth of untreated B. amyloliquefaciens spores was $6 \%$. Hence, both non-injured and injured spores would have the capacity to form colonies on nutrient agar medium while only non-injured spores could from colonies on the selective medium. Colonies were counted with a colony counter (Schuett-biotec, Göttingen, Germany). All inactivation graphs were obtained with Microcal Origin 8.0 (Microcal Software, Inc., Northampton, USA).

\section{Assessment of Inactivation Kinetic Parameters}

The inactivation of microorganisms has frequently been described by a first-order reaction. The reduction of microorganisms could be expressed as Equation (1) at a given temperature, where $\mathrm{N}_{0}$ is the initial spore concentration, and $\mathrm{N}_{\mathrm{t}}$ is the corresponding viable number of spores at time t. The decimal reduction time $\mathrm{D}$ is the time required at a certain temperature to kill $90 \%$ of the microorganisms. It can be calculated from the semi logarithmic inactivation profile. The $z$-value is the temperature increase required to reduce the $\mathrm{D}$ value to a tenth of the original value. The rate constant $\mathrm{k}$ is obtained by Equation (2). The Arrhenius Equation (3) indicated the temperature dependence of the inactivation rate constant $\mathrm{k}$.

However, thermal inactivation kinetics of B. amyloliquefaciens spores showed an initial lag phase ("shoulder") which could not be sufficiently described by Equation (1), even when considering potentially different orders (Van Boekel, 2010). Another approach to model shoulder formations by assuming a non cultivable fraction of the initial population which would be activated by thermal shocks was also rejected (Abraham et al., 1990; Kessler, 2002). Based on the kinetics in this work, it would imply a very high speculated initial non cultivable population which was not observed by phase contrast microscopy. Thus, a Weibull model was also considered to assess the thermal inactivation kinetics. As shown in Equation (4), b is the scale factor and $\mathrm{n}$ is the shape factor (Peleg and Cole, 1998). The $\mathrm{n}$ parameter was not, or only slightly, dependent on temperature. It could be assumed as constant with varying temperatures, and an average value was calculated for modeling, which was 2.24, 2.22, and 1.84 for PBS, low fat milk, and whole milk, respectively. The temperature dependence of $b$ could be expressed as the log-logistic model Equation (5), where $\mathrm{T}_{\mathrm{c}}$ is the lethal temperature marker representing the temperature range where the inactivation accelerates and the lethality rate factor $\mathrm{k}$ is the rate representing the increase of $\mathrm{b}$ with temperature. This model was suggested by Corradini et al. (2005) and Periago et al. (2004) following work with Clostridium botulinum 213B and Bacillus sporothermodurans IC4 spores suspended in PBS and soups and was validated for thermal inactivation in the range of $101-125^{\circ} \mathrm{C}$. According to this model, when $\mathrm{T} \ll \mathrm{Tc}, \mathrm{b} \approx 0$ and when $\mathrm{T} \gg \mathrm{Tc}, \mathrm{b} \approx \mathrm{k}(\mathrm{T}-\mathrm{Tc}$ ) (Van Boekel, 2002).

The adjusted $\mathrm{R}^{2}$ given by Equation (6) is a modification of the correlation coefficient $\left(\mathrm{R}^{2}\right)$. It indicates how well data points fit the curve, but it is adjusted for the number of parameters in a model and thus gives a more reasonable test of fit than $\mathrm{R}^{2}$ or the mean square error (MSE), especially when there are few data ( $n$, number of data points) or a great number of parameters $\left(\mathrm{N}_{\mathrm{T}}\right)$ in the model (Davey, 1993; Khoo et al., 2003). When $n \gg N_{T}$, adjusted $R^{2}$ approached the value of $R^{2}$. The adjusted $\mathrm{R}^{2}$ allows for a comparative assessment of models with different numbers of parameters, which is important in this study.

$$
\log \left(\frac{\mathrm{N}_{\mathrm{t}}}{\mathrm{N}_{0}}\right)=-\mathrm{t} \cdot \frac{\mathrm{k}}{2.303}
$$




$$
\begin{gathered}
\mathrm{D}=\frac{2.303}{\mathrm{k}} \\
\ln \left(\frac{\mathrm{k}}{\mathrm{k}_{0}}\right)=-\frac{\mathrm{E}_{\mathrm{a}}}{\mathrm{R}} \cdot \frac{1}{\mathrm{~T}} \\
\log \left(\frac{\mathrm{N}_{\mathrm{t}}}{\mathrm{N}_{0}}\right)=-\mathrm{b} \cdot t^{n} \\
\mathrm{~b}=\ln \left[1+\exp \left(\mathrm{k}\left(\mathrm{T}-\mathrm{T}_{\mathrm{c}}\right)\right)\right] \\
\text { Adjusted } \mathrm{R}^{2}=1-\frac{\left(1-\mathrm{R}^{2}\right)(\mathrm{n}-1)}{\left(\mathrm{n}-\mathrm{N}_{\mathrm{T}}-1\right)}
\end{gathered}
$$

\section{Results}

\section{Assessment of Thermal Inactivation Kinetics}

The thermal inactivation kinetics B. amyloliquefaciens spores in PBS and milk at $105,110,115$, and $121^{\circ} \mathrm{C}$ are shown in Figure 2, with detailed kinetic parameters in Table 2. The decimal reduction time $\mathrm{D}, z$-value and energy of activation $\mathrm{E}_{\mathrm{a}}$ were evaluated based on the first-order reaction model.

A higher D value was found for spores in milk vs. PBS at $105^{\circ} \mathrm{C}$, while at 110,115 , and $121^{\circ} \mathrm{C}$, the D values in PBS or milk were not significantly different. The $z$-values of spores in PBS, lower fat milk, and whole milk were $7.02,6.28$, and $6.03^{\circ} \mathrm{C}$, respectively. The $E_{a}$ increased from $406.18 \mathrm{~kJ} / \mathrm{mol}$ in PBS to $473.35 \mathrm{~kJ} / \mathrm{mol}$ in whole milk, which suggested that the milk fat had some protective effect against thermal inactivation. One should however bear in mind that the overall fitting of the firstorder reaction model to the experimental data was not optimal. In this sense, Weibull modeling offered a much better fitting. The adjusted $\mathrm{R}^{2}$ was determined to evaluate the accuracy of fitting models and showed that the use of a nonlinear model to describe $B$. amyloliquefaciens spore thermal inactivation is more appropriate from a statistical point of view. The $b$ values increased with the increase of temperature, while the $n$ values were slightly dependent on the temperature. Based on Equations (4) and (5), the b and n parameters were evaluated (Figure 3) and the modeled inactivation under higher temperatures, as such reached during UHPH, was calculated. There could be a risk of deviation from the real data in the extrapolation and it must be taken cautiously. However, it is important to point out that determination of the inactivation at $T>121^{\circ} \mathrm{C}$ was impossible experimentally because the very short holding times $(<1 \mathrm{~s})$ required to quantify the inactivation vs. time would not allow to reach the target temperature within the glass capillaries before full inactivation occurs (Mathys et al., 2007b).

\section{UHPH Inactivation vs. Pressure and Valve Temperature}

The inactivation of B. amyloliquefaciens spores in PBS and milk were plotted vs. the pressure (Figure 4) and the valve temperature (Figure 5). The valve temperature increased linearly with the increase of homogenization pressure at constant inlet temperature. This increase was the consequence of the intense energy conversion occurring at the first valve involving kinetic energy, turbulence, shear and cavitation forces (Hayes and Kelly, 2003; Thiebaud et al., 2003; Zamora and Guamis, 2015). It was reported that an increase of $22^{\circ} \mathrm{C} / 100 \mathrm{MPa}$ could be achieved at the valve of Stansted Fluid Power UHPH systems (Floury et al., 2004). In this study, temperature increase during UHPH treatments was similar and no significant difference was found between PBS buffer and milk.

As shown in Figure 4, the inactivation of B. amyloliquefaciens spores was enhanced with the increased pressure. No reduction of B. amyloliquefaciens spores was found at $200 \mathrm{MPa}$. Up to $3.5 \log _{10} \mathrm{CFU} / \mathrm{mL}$ reduction of spores was achieved at $350 \mathrm{MPa}$. The residence time after valve at high temperature and before cooling was estimated at $0.24 \mathrm{~s}$ based on the measured flow rate and equipment dimensions. Based on the experimental thermal inactivation of spores and extrapolation, different potential residence times ranging from 0.24 to $0.54 \mathrm{~s}$ were chosen to estimate the thermal inactivation cause by valve temperature during UHPH treatments. This range was selected to simulate potential deviations from the estimated mean residence time which are frequently reported for continuous processes. As shown in Figure 5, the increment in the valve temperature increased the spore inactivation. A valve temperature lower than $125^{\circ} \mathrm{C}$ during treatment at $200 \mathrm{MPa}$ with an inlet temperature of $80^{\circ} \mathrm{C}$ led to no reduction of spores. With the increase of pressure, the valve temperature also increased and a higher spore inactivation was achieved. A reduction of $3.5 \log _{10} \mathrm{CFU} / \mathrm{mL}$ of $B$. amyloliquefaciens spores was achieved by treatment at $350 \mathrm{MPa}$ with a valve temperature higher than $150^{\circ} \mathrm{C}$ and a very short residence time.

\section{Discussion}

\section{Impact of the Homogenization Pressure on B. amyloliquefaciens Spore Inactivation}

Based on Figure 4, it could be tempting to conclude that there is a linear correlation between homogenization pressure and inactivation. However, while this holds for high inlet temperatures, previous work (Georget et al., 2014a) also found that for lower inlet temperatures no inactivation could be achieved even for pressures as high as $350 \mathrm{MPa}$. Hence, the homogenization pressure appears to be a necessary facilitator for UHPH spore inactivation, but needs to be combined to high inlet and valve temperatures to yield a sufficient inactivation effect.

\section{Impact of the Valve Temperature on $B$. amyloliquefaciens Spore Inactivation}

Inactivation of bacterial spores has commonly been modeled by a first-order reaction, even though a nonlinear inactivation curve was often observed. In this work, both log-linear and Weibull models were used to assess the thermal inactivation kinetics of $B$. amyloliquefaciens spores. Strong deviations to linear inactivation kinetics were observed and Weibull modeling provided a much better fit of experimental data, as was verified by the adjust $\mathrm{R}^{2}$ and could not be replaced by conventionally suggested alternative models (see Section Assessment of Inactivation Kinetic Parameters). Based on Weibull modeling and the determination of the temperature dependency of b Equation (5), the predicted thermal inactivation at very high temperatures/short holding times could be 

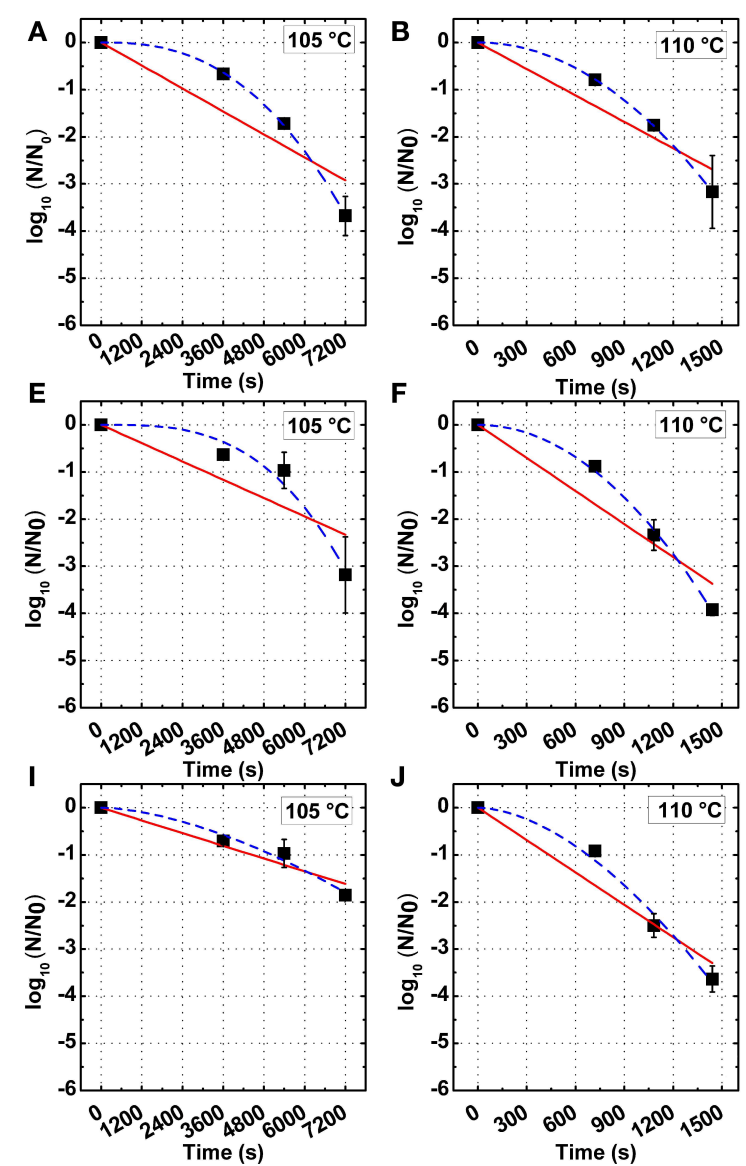
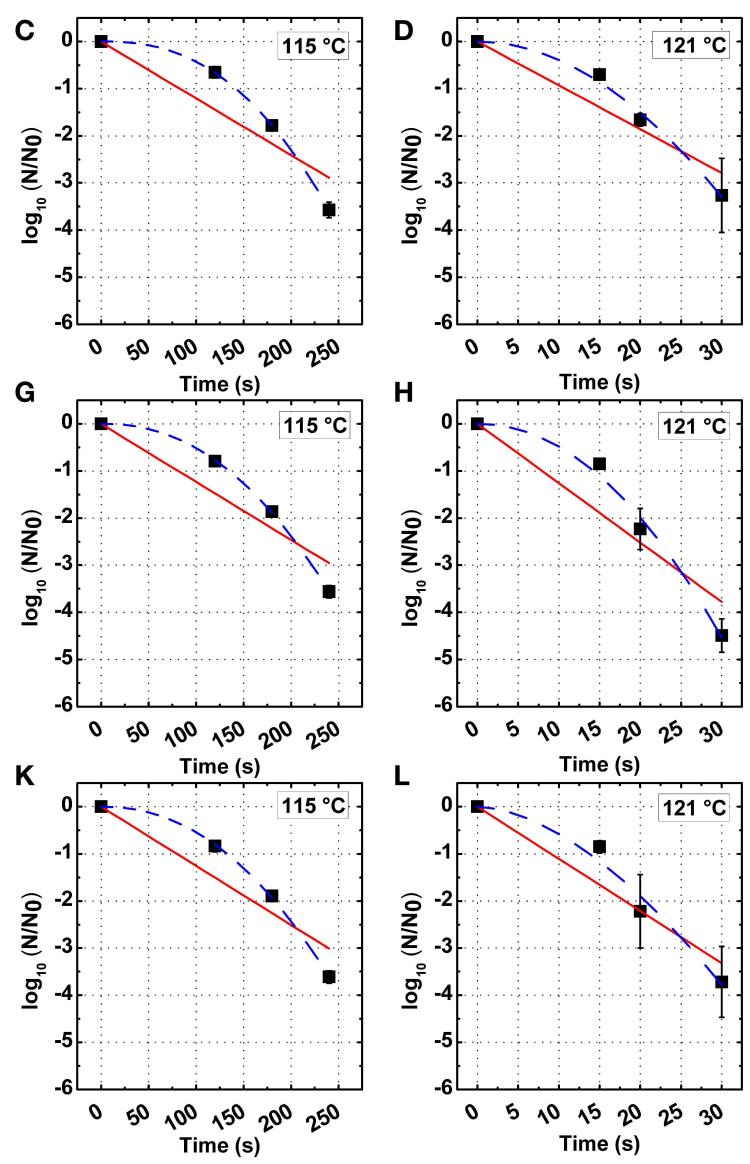

FIGURE 2 | First-order (red continuous line) and Weibull (blue dashed line) model of the inactivation kinetics of B. amyloliquefaciens spores in PBS (A-D), low fat milk (E-H), and whole milk (I-L) at 105, 110, 115, and $121^{\circ} \mathrm{C}$.

extrapolated to estimate the thermal contribution during UHPH treatments.

Previous studies showed that the inactivation of spores by UHPH increased with the increase of the inlet temperature. For G. stearothermophilus ATCC7953 and C. sporogenes PA3679 spores in skim milk, UHPH treatment of 16 passes at $300 \mathrm{MPa}$ with inlet temperature at $45^{\circ} \mathrm{C}$ caused a reduction of only 0.67 $\log _{10} \mathrm{CFU} / \mathrm{mL}$ (Pinho et al., 2011). No reduction of B. subtilis and G. stearothermophilus ATCC7953 spores was observed after treatment at 300 and $350 \mathrm{MPa}$ with the inlet temperature at 37 and $55^{\circ} \mathrm{C}$ respectively. However, with inlet temperatures of $80^{\circ} \mathrm{C}$, up to $5 \log _{10} \mathrm{CFU} / \mathrm{mL}$ B. subtilis PS832 spores and $2 \log _{10} \mathrm{CFU} / \mathrm{mL}$ G. stearothermophilus ATCC7953 spores were inactivated at $350 \mathrm{MPa}$ (Georget et al., 2014a). This latter result also shows that the stronger resistance of a thermophilic strain and indicator for wet heat sterilization (G. stearothermophilus ATCC7953) correlated to its lesser inactivation by UHPH in comparison to a mesophilic strain such as $B$. subtilis PS832. It was also reported that treatment at $300 \mathrm{MPa}$ with the inlet temperature at $85^{\circ} \mathrm{C}$ could completely inactivate $B$. cereus, B. licheniformis, B. sporothermodurans, B. coagulans, $G$. stearothermophilus, and B. subtilis spores in whole milk at the inoculating level of $6 \log _{10} \mathrm{CFU} / \mathrm{mL}$. The $B$. cereus spores were below the detection limit for treatment at $200 \mathrm{MPa}$ with the inlet temperature at $75^{\circ} \mathrm{C}$ and treatments at $300 \mathrm{MPa}$ with inlet temperature at 55,65 , and $75^{\circ} \mathrm{C}$ (Valencia-Flores et al., 2013).

In this work, the overlapping of the predicted thermal inactivation kinetics and the experimentally observed UHPH inactivation indicated that in spite of the very short holding time before cooling takes place, the temperatures reached are high enough to induce significant thermal inactivation. The overlap of the predicted thermal and UHPH inactivation profiles suggests that the achieved inactivation of $B$. amyloliquefaciens spores was slightly higher than predicted based on thermal only inactivation with the estimated residence time of $0.24 \mathrm{~s}$. UHPH inactivation profiles were closer to thermal inactivation profiles modeled with residence times of $0.36,0.48$, and $0.30 \mathrm{~s}$ in PBS, low fat milk, and whole milk, respectively. This could mean that either mechanic stresses at the valve additionally improved the inactivation post valve or that deviations to the estimated mean residence time occurred as can be observed in continuous systems with complex flow patterns (Georget et al., 2013). This latter hypothesis seems plausible, in particular when considering the very small range of residence times considered here $(0.24-0.54 \mathrm{~s})$. The modeled 
TABLE 2 | First-order and Weibull thermal inactivation models' kinetic parameters ( \pm followed by corresponding standard deviation).

\begin{tabular}{|c|c|c|c|c|c|c|c|c|}
\hline Medium & Temperature $\left({ }^{\circ} \mathrm{C}\right)$ & \multicolumn{4}{|c|}{ First-order model } & \multicolumn{3}{|c|}{ Weibull model } \\
\hline \multirow{3}{*}{ PBS } & 110 & $542.69 \pm 77.28$ & & & 0.94176 & $(1.09 \pm 1.08) \times 10^{-5}$ & $2.01 \pm 0.61$ & 0.99987 \\
\hline & 115 & $94.29 \pm 19.40$ & & & 0.89894 & $(1.29 \pm 1.67) \times 10^{-5}$ & $2.45 \pm 0.42$ & 0.99998 \\
\hline & 121 & $11.90 \pm 2.12$ & & & 0.92851 & $(4.47 \pm 2.54) \times 10^{-3}$ & $1.96 \pm 0.25$ & 0.98724 \\
\hline & 110 & $427.63 \pm 24.07$ & & & 0.93829 & $(2.01 \pm 1.10) \times 10^{-6}$ & $2.01 \pm 0.09$ & 0.99560 \\
\hline & 115 & $81.28 \pm 2.83$ & & & 0.92379 & $(2.27 \pm 1.36) \times 10^{-5}$ & $2.20 \pm 0.11$ & 0.99978 \\
\hline & 121 & $7.97 \pm 0.63$ & & & 0.91390 & $(5.51 \pm 4.88) \times 10^{-3}$ & $2.05 \pm 0.26$ & 0.98350 \\
\hline Whole milk & 105 & $4474.11 \pm 418.34$ & $6.03 \pm 0.10$ & $473.35 \pm 7.97$ & 0.96538 & $(0.89 \pm 1.51) \times 10^{-5}$ & $1.72 \pm 0.45$ & 0.96463 \\
\hline
\end{tabular}

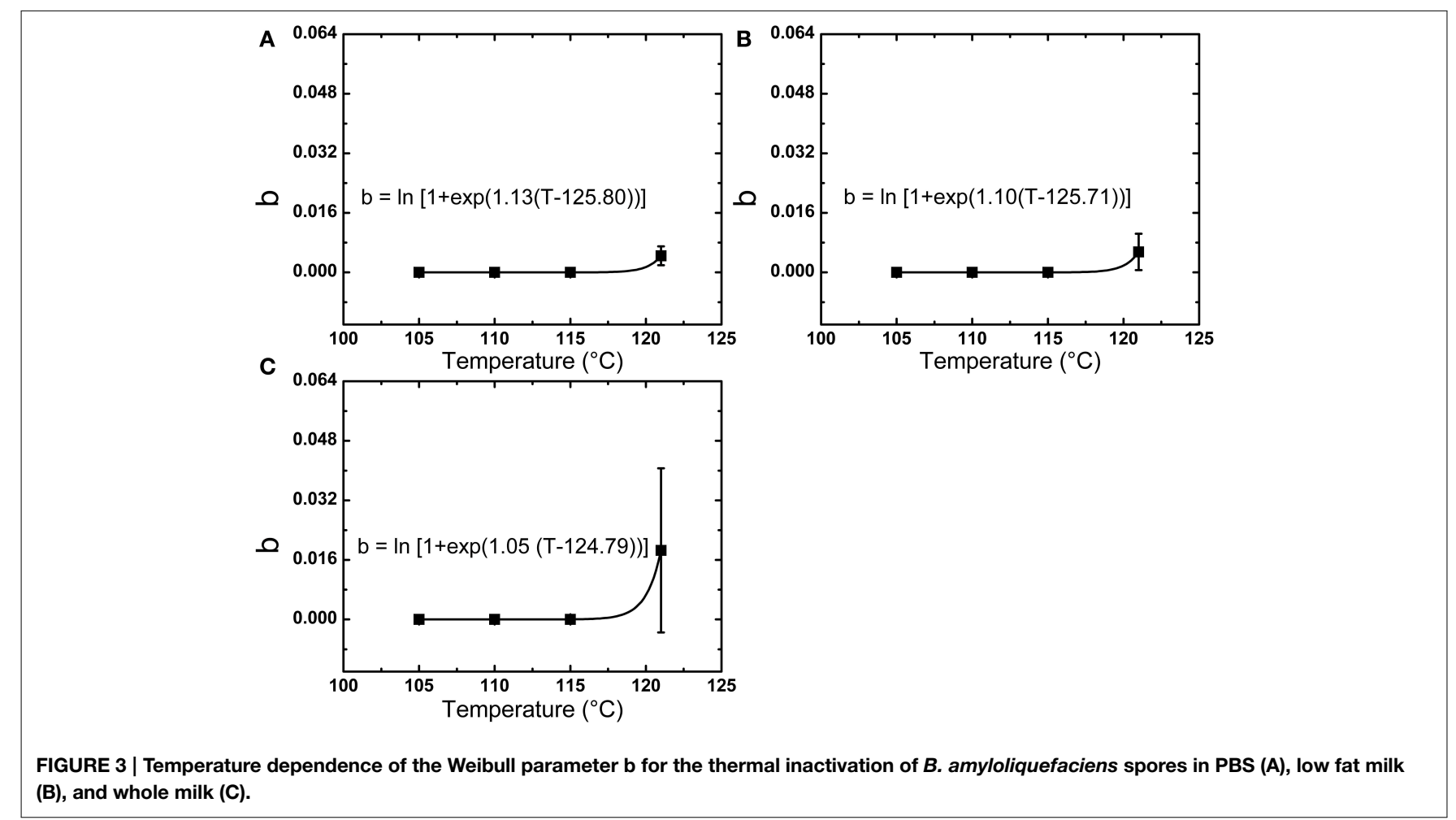

thermal inactivation profiles further emphasize the potential impact of very high temperatures on inactivation kinetics and the strong dependence on residence times, even for very short ones.

Previous work suggested that when valve temperatures below $60^{\circ} \mathrm{C}$ were reached during UHPH, microbial inactivation could be a synergetic action of all stress factors including cavitation, shear stress, turbulence, impingement and high pressure. However, when valve temperatures reached $80^{\circ} \mathrm{C}$ or above, the observed microbial inactivation was mainly caused by thermal effects (Pathanibul et al., 2009; Dumay et al., 2013;
Georget et al., 2014b). This would also support the second hypothesis that deviations to the mean residence time might explain the slightly stronger spore inactivation observed by UHPH. Local recirculation zones before the cooling section could contribute to this behavior. Therefore, a proper characterization of the residence time distribution and temperature profiles for the considered valve design by computational fluid dynamic could increase the understanding of the key process parameters and potentially confirm the dominant role of temperature in bacterial spore inactivation by UHPH. 

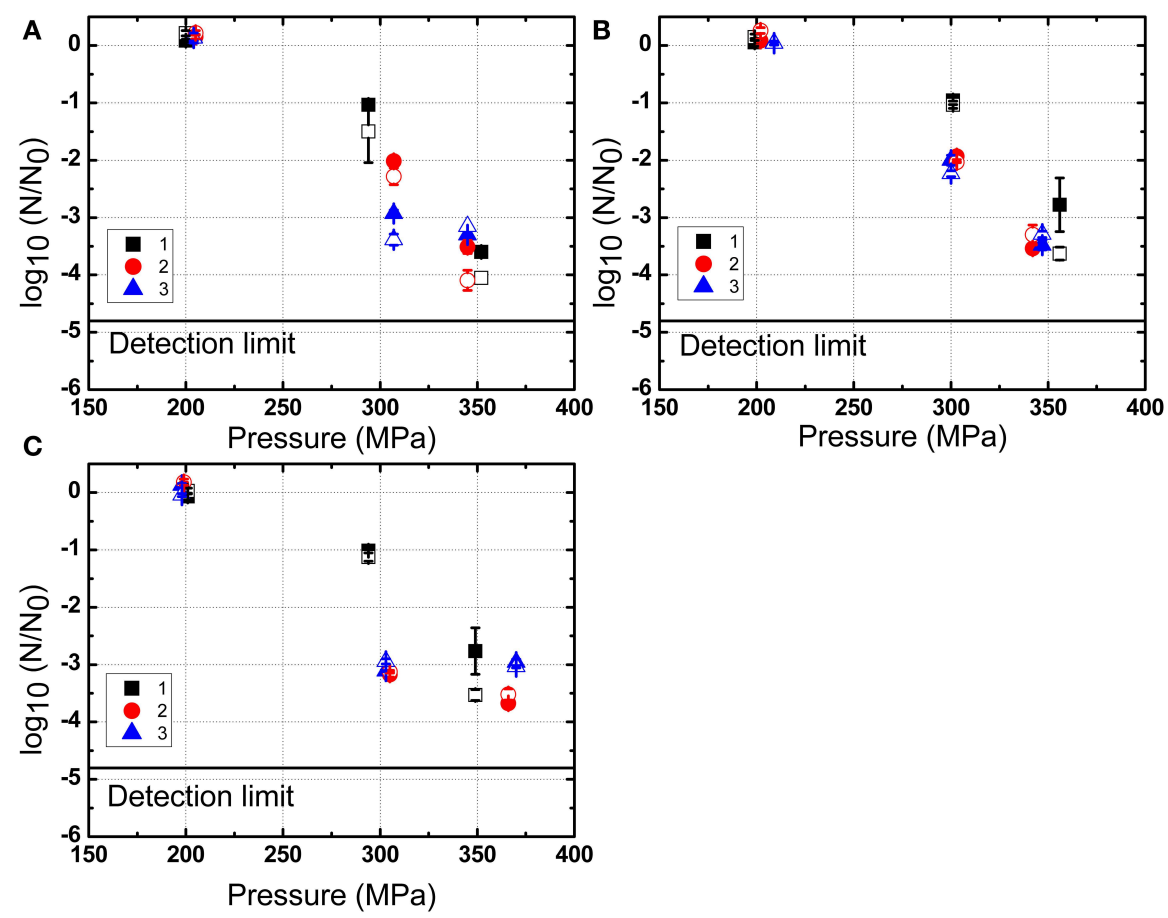

FIGURE 4 | Inactivation of $B$. amyloliquefaciens spores in PBS (A), low fat milk (B), and whole milk (C) as a function of the pressure. 1, 2, and 3 represent three independent repetitions of the trial with the same initial batch of spores. The solid symbols represent results obtained by plating on nutrient agar medium and the corresponding hollow symbols are based on the use of the selective medium. The data shown are independent from the valve temperature.
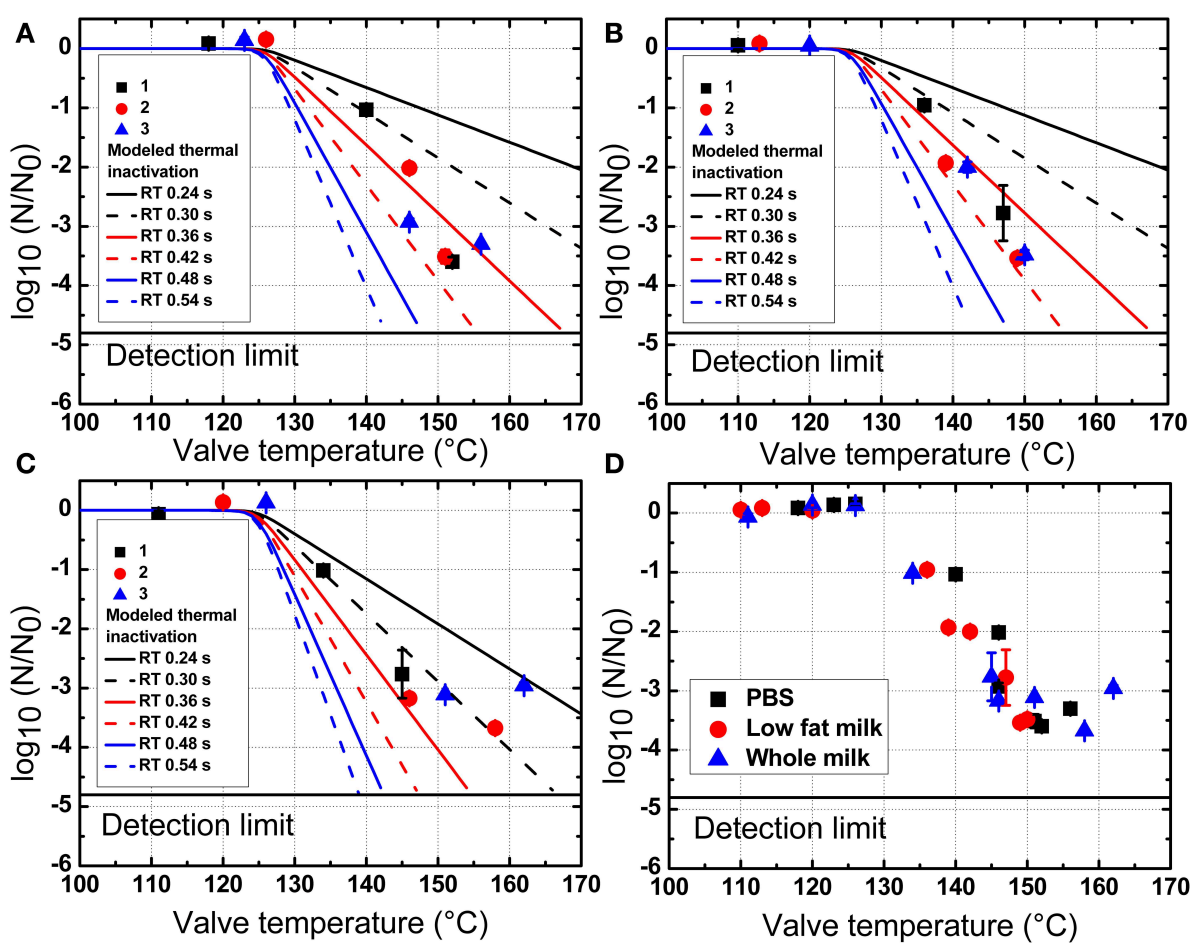

D

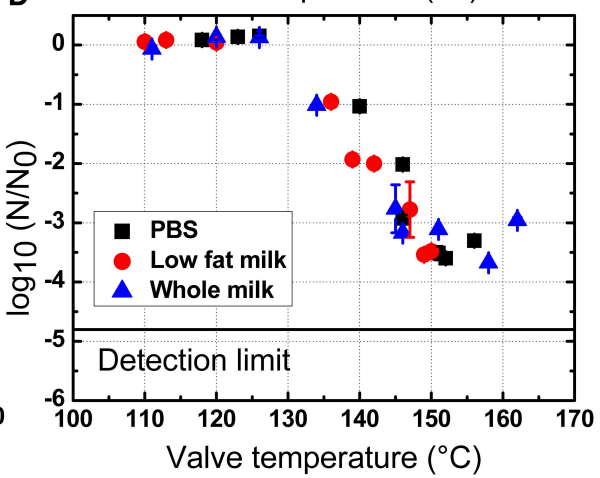

FIGURE 5 | Inactivation of B. amyloliquefaciens spores in PBS (A), low fat milk (B), and whole milk (C) as a function of the valve temperature. All experimental plots are summarized in (D). 1, 2, and 3 represent three independent repetitions of the trial with the same initial batch of spores. The modeled thermal inactivations are based on $n$ values at 2.24 (A), 2.22 (B), and 1.84 (C) and b values calculated by equations in Figure 3. The data shown are independent from the homogenization pressure. 


\section{Sublethal Injury of B. amyloliquefaciens Spores}

No significant difference was observed in the colonies between selective and non selective media (Figure 4), indicating that the UHPH treatment apparently caused no sublethal injured spores. Inactivation by UHPH was often regarded as an "all or nothing" mechanism, which means no sublethal injury to the cells (Donsì et al., 2009). If the cells are disrupted into small fragments by the mechanical stresses, they are dead, otherwise, they are alive. There is no other physiological state in between. Existing studies found that no sublethal injury was caused in vegetative microorganisms by UHPH. Yersinia enterocolitica and Staphylococcus aureus were treated at 200 and $300 \mathrm{MPa}$ with the inlet temperature at $25^{\circ} \mathrm{C}$, and no sublethal injury was observed using low $\mathrm{pH}(5.5-7), \mathrm{NaCl}(0-6 \%)$ or sodium dodecyl sulfate (SDS, $0-100 \mathrm{mg} / \mathrm{L}$ ) as selective components in the plate medium. These findings differed from the high isostatic pressure processing that could result in an accumulation of sublethal injury, eventually leading to inactivation (Wuytack et al., 2002). Similar results were also found in UHPH treated L. monocytogenens in milk as well as S. aureus and S. carnosus inoculated in milk and orange juice (Briñez et al., 2007; RoigSagués et al., 2009). The same conclusion might apply to bacterial spores which are furthermore protected against mechanical stresses by their multi-layered and highly condensed structure. Hence, the inactivation mechanism in the matrices considered might be a binary one-once a certain stress threshold is reached, the spores are inactivated, and otherwise they stay physiologically fit.

\section{Protective Effect of Milk Fat during UHPH Treatments}

Characteristics of food matrices (water and fat contents, viscosity, $\mathrm{pH}$, etc.) have been found to influence microbial inactivation during UHPH treatments (Dumay et al., 2013). Some authors reported the milk fat could have a protective influence on vegetative microorganisms during UHPH treatments, in analogy to the well-established protective effect of milk fat during thermal treatments (MacDonald and Sutherland, 1993). Others suggested that fat might induce locally a stronger heating and thus improve inactivation (Roig-Sagués et al., 2009). UHPH treatments at $200 \mathrm{MPa}$ with an inlet temperature at $28^{\circ} \mathrm{C}$ for 51 -min cycles resulted in a reduction of 2,3 , and $4 \log _{10} \mathrm{CFU} / \mathrm{mL}$ of endogenous flora in full fat $(3.5 \%)$, low fat (2\%), and skim milk, respectively (Kheadr et al., 2002). The degree of inactivation of L. monocytogenes in milk was significantly lower than in PBS (Vachon et al., 2002). Milk being an oil-in-water emulsion, this protective effect could

\section{References}

Abraham, G., Debray, E., Candau, Y., and Piar, G. (1990). Mathematical model of thermal destruction of Bacillus stearothermophilus spores. Appl. Environ. Microbiol. 56, 3073-3080.

Amador Espejo, G. G., Hernández-Herrero, M. M., Juan, B., and Trujillo, A. J. (2014). Inactivation of Bacillus spores inoculated in milk by ultra high pressure homogenization. Food Microbiol. 44, 204-210. doi: 10.1016/j.fm.2014.06.010 be explained by the interactions between fat globules and microorganisms. The size of fat globules decreased with the increase of homogenization pressure during treatments, and the particle size distribution was shifted toward smaller values. The volume weighted mean value $\mathrm{D}[4,3]$ decreased from $3.81 \mathrm{~mm}$ (control raw milk) to $0.189 \mathrm{~mm}$ after homogenization at $300 \mathrm{MPa}$ (Picart et al., 2006; Dumay et al., 2013). Fat globules could offer local low water activity refuges to the microorganisms and thus provide potential protective effect against thermal inactivation.

However, contrasting results were also reported with the higher inactivation of $E$. coli in whole milk than in PBS and skim milk (Vachon et al., 2002; Briñez et al., 2006). It was also found that fat content increased the inactivation of L. monocytogenes in milk (Roig-Sagués et al., 2009). By evaluating the inactivation of $E$. coli in buffer solutions with different viscosities and food systems (skim milk, soy milk, and strawberry-raspberry milk), Diels et al. (2005) found that the inactivation of E. coli by UHPH was influenced by fluid viscosity, which was against the protective effect of milk fat.

In the present work, all experimental plots of $B$. amyloliquefaciens spores in PBS, low fat milk, and whole milk as a function of valve temperature are shown in Figure 5D. It could be observed that the milk fat had no clear protective effect on spores against UHPH treatments.

In conclusion, UHPH could be a promising alternative to high temperature sterilization for the preservation of pumpable and particle free foods. The modeled thermal inactivation matching UHPH treatment parameters suggests that the observed reduction in spore counts could be temperature driven in agreement with previous work by the authors. A reduction of $3.5 \log _{10} \mathrm{CFU} / \mathrm{mL}$ of $B$. amyloliquefaciens spores was achieved at $350 \mathrm{MPa}$ with the valve temperature higher than $150^{\circ} \mathrm{C}$ for very short holding times. No sublethal injury was observed after UHPH treatments using sodium chloride as selective component in the nutrient agar medium. Future work based on advanced fluid dynamic modeling including exact residence time and temperature distributions could also allow refining the approach used in this work and assess whether stress factors such as turbulences or shear stresses could additionally contribute to thermal inactivation during UHPH.

\section{Acknowledgments}

The author PD would like to acknowledge China Scholarship Council (CSC No. 201406350127). and modeling kinetics of Alicyclobacillus acidoterrestris spores in white grape and apple juices. Int. J. Food Microbiol. 166, 494-498. doi: 10.1016/j.ijfoodmicro.2013.08.015

Briñez, W. J., Roig-Sagués, A. X., Hernández-Herrero, M. M., and Guamis-López, B. (2006). Inactivation of two strains of Escherichia coli inoculated into whole and skim milk by ultra high-pressure homogenisation. Lait 86, 241-249. doi: 10.1051/lait:2006006 
Briñez, W. J., Roig-Sagués, A. X., Hernández-Herrero, M. M., and GuamisLópez, B. (2007). Inactivation of Staphylococcus spp. strains in whole milk and orange juice using ultra high pressure homogenisation at inlet temperatures of 6 and $20^{\circ}$ C. Food Control 18, 1282-1288. doi: 10.1016/j.foodcont.2006. 09.002

Burston, H. E., Davey, M., and Conibear, E. (2008). "Genome-wide analysis of membrane transport using yeast knockout arrays," in Methods in Molecular Biology, Membrane Trafficking, ed A. Vancura (New York, NY: Humana Press), 29-39.

Corradini, M. G., Normand, M. D., and Peleg, M. (2005). Calculating the efficacy of heat sterilization processes. J. Food Eng. 67, 59-69. doi: 10.1016/j.jfoodeng.2004.08.001

Davey, K. R. (1993). Linear-Arrhenius models for bacterial growth and death and vitamin denaturations. J. Ind. Microbiol. 12, 172-179. doi: 10.1007/BF01584187

Diels, A. M., Callewaert, L., Wuytack, E. Y., Masschalck, B., and Michiels, C. W. (2005). Inactivation of Escherichia coli by high-pressure homogenisation is influenced by fluid viscosity but not by water activity and product composition. Int. J. Food Microbiol. 101, 281-291. doi: 10.1016/j.ijfoodmicro.2004.11.011

Diels, A. M., and Michiels, C. W. (2006). High-pressure homogenization as a nonthermal technique for the inactivation of microorganisms. Crit. Rev. Microbiol. 32, 201-216. doi: 10.1080/10408410601023516

Donsì, F., Ferrari, G., Lenza, E., and Maresca, P. (2009). Main factors regulating microbial inactivation by high-pressure homogenization: operating parameters and scale of operation. Chem. Eng. Sci. 64, 520-532. doi: 10.1016/j.ces.2008.10.002

Dumay, E., Chevalier-Lucia, D., Picart-Palmade, L., Benzaria, A., Gràcia-Julià, A., and Blayo, C. (2013). Technological aspects and potential applications of (ultra) high-pressure homogenisation. Trends Food Sci. Technol. 31, 13-26. doi: 10.1016/j.tifs.2012.03.005

Floury, J., Bellettre, J., Legrand, J., and Desrumaux, A. (2004). Analysis of a new type of high pressure homogeniser. A study of the flow pattern. Chem. Eng. Sci. 59, 843-853. doi: 10.1016/j.ces.2003.11.017

Gayán, E., Álvarez, I., and Condón, S. (2013). Inactivation of bacterial spores by UV-C light. Innov. Food Sci. Emerg. Technol. 19, 140-145. doi: 10.1016/j.ifset.2013.04.007

Georget, E., Miller, B., Aganovic, K., Callanan, M., Heinz, V., and Mathys, A. (2014a). Bacterial spore inactivation by ultra-high pressure homogenization. Innov. Food Sci. Emerg. Technol. 26, 116-123. doi: 10.1016/j.ifset.2014.08.004

Georget, E., Miller, B., Callanan, M., Heinz, V., and Mathys, A. (2014b). (Ultra) high pressure homogenization for continuous high pressure sterilization of pumpable foods-a review. Front. Nutr. 1:15. doi: 10.3389/fnut.2014.00015

Georget, E., Sauvageat, J. L., Burbidge, A., and Mathys, A. (2013). Residence time distributions in a modular micro reaction system. J. Food Eng. 116, 910-919. doi: 10.1016/j.jfoodeng.2013.01.041

Georget, E., Sevenich, R., Reineke, K., Mathys, A., Heinz, V., Callanan, M., et al. (2015). Inactivation of microorganisms by high isostatic pressure processing in complex matrices: a review. Innov. Food Sci. Emerg. Technol. 27, 1-14. doi: $10.1016 /$ j.ifset.2014.10.015

Hayes, M. G., and Kelly, A. L. (2003). High pressure homogenisation of raw whole bovine milk (a) effects on fat globule size and other properties. J. Dairy Res. 70, 297-305. doi: 10.1017/S0022029903006320

Kessler, H. G. (2002). Food and Bio Process Engineering: Dairy Technology, 5th Edn. Munich: Publishing House A. Kessler.

Kheadr, E. E., Vachon, J. F., Paquin, P., and Fliss, I. (2002). Effect of dynamic high pressure on microbiological, rheological and microstructural quality of Cheddar cheese. Int. Dairy J. 12, 435-446. doi: 10.1016/S0958-6946(01)00104-2

Khoo, K. Y., Davey, K. R., and Thomas, C. J. (2003). Assessment of four model forms for predicting thermal inactivation kinetics of Escherichia coli in liquid as affected by combined exposure time, liquid temperature and pH. Food Bioprod. Process. 81, 129-137. doi: 10.1205/096030803322088260

MacDonald, F., and Sutherland, A. D. (1993). Effect of heat treatment on Listeria monocytogenes and gram-negative bacteria in sheep, cow and goat milks. J. Appl. Bacteriol. 75, 336-343. doi: 10.1111/j.1365-2672.1993.tb0 2785. $\mathrm{x}$

Margosch, D., Ehrmann, M. A., Buckow, R., Heinz, V., Vogel, R. F., and Gänzle, M. G. (2006). High-pressure-mediated survival of Clostridium botulinum and Bacillus amyloliquefaciens endospores at high temperature. Appl. Environ. Microbiol. 72, 3476-3481. doi: 10.1128/AEM.72.5.3476-3481.2006
Mathys, A. (2008). Inactivation Mechanisms of Geobacillus and Bacillus Spores during High Pressure Thermal Sterilization. Ph.D. dissertation, Technische Universität Berlin.

Mathys, A., Chapman, B., Bull, M., Heinz, V., and Knorr, D. (2007a). Flow cytometric assessment of Bacillus spore response to high pressure and heat. Innov. Food Sci. Emerg. Technol. 8, 519-527. doi: 10.1016/j.ifset.2007. 06.010

Mathys, A., Heinz, V., Schwartz, F. H., and Knorr, D. (2007b). Impact of agglomeration on the quantitative assessment of Bacillus stearothermophilus heat inactivation. J. Food Eng. 81, 380-387. doi: 10.1016/j.jfoodeng.2006.11.012

Pathanibul, P., Taylor, T. M., Davidson, P. M., and Harte, F. (2009). Inactivation of Escherichia coli and Listeria innocua in apple and carrot juices using high pressure homogenization and nisin. Int. J. Food Microbiol. 129, 316-320. doi: 10.1016/j.ijfoodmicro.2008.12.020

Peleg, M., and Cole, M. B. (1998). Reinterpretation of microbial survival curves. Crit. Rev. Food Sci. Nutr. 38, 353-380. doi: 10.1080/10408699891274246

Pereda, J., Ferragut, V., Quevedo, J. M., Guamis, B., and Trujillo, A. J. (2007). Effects of ultra-high pressure homogenization on microbial and physicochemical shelf life of milk. J. Dairy Sci. 90, 1081-1093. doi: 10.3168/jds.S0022-0302(07)71595-3

Periago, P. M., van Zuijlen, A., Fernandez, P. S., Klapwijk, P. M., ter Steeg, P. F., Corradini, M. G., et al. (2004). Estimation of the non-isothermal inactivation patterns of Bacillus sporothermodurans IC4 spores in soups from their isothermal survival data. Int. J. Food Microbiol. 95, 205-218. doi: 10.1016/j.ijfoodmicro.2004.02.015

Picart, L., Thiebaud, M., René, M., Pierre Guiraud, J., Cheftel, J. C., and Dumay, E. (2006). Effects of high pressure homogenisation of raw bovine milk on alkaline phosphatase and microbial inactivation. A comparison with continuous short-time thermal treatments. J. Dairy Res. 73, 454-463. doi: $10.1017 /$ S0022029906001853

Pinho, C. R. G., Franchi, M. A., Tribst, A. A. L., and Cristianinia, M. (2011). Effect of high pressure homogenization process on Bacillus stearothermophilus and Clostridium sporogenes spores in skim milk. Procedia Food Sci. 1, 869-873. doi: 10.1016/j.profoo.2011.09.131

Reineke, K., Mathys, A., Heinz, V., and Knorr, D. (2013). Mechanisms of endospore inactivation under high pressure. Trends Microbiol. 21, 296-304. doi: 10.1016/j.tim.2013.03.001

Reineke, K., Mathys, A., and Knorr, D. (2011a). The impact of high pressure and temperature on bacterial spores: inactivation mechanisms of Bacillus subtilis above 500 MPa. J. Food Sci. 76, M189-M197. doi: 10.1111/j.17503841.2011.02066.x

Reineke, K., Mathys, A., and Knorr, D. (2011b). Shift of pH-value during thermal treatments in buffer solutions and selected foods. Int. J. Food Prop. 14, 870-881. doi: 10.1080/10942910903456978

Roig-Sagués, A. X., Velázquez, R. M., Montealegre-Agramont, P., LópezPedemonte, T. J., Briñez-Zambrano, W. J., Guamis-López, B., et al. (2009). Fat content increases the lethality of ultra-high-pressure homogenization on Listeria monocytogenes in milk. J. Dairy Sci. 92, 5396-5402. doi: $10.3168 /$ jds.2009-2495

Sevenich, R., Bark, F., Crews, C., Anderson, W., Pye, C., Riddellova, K., et al. (2013). Effect of high pressure thermal sterilization on the formation of food processing contaminants. Innov. Food Sci. Emerg. Technol. 20, 42-50. doi: 10.1016/j.ifset.2013.07.006

Siemer, C., Toepfl, S., and Heinz, V. (2014a). Inactivation of Bacillus subtilis spores by pulsed electric fields (PEF) in combination with thermal energy I. Influence of process- and product parameters. Food Control 39, 163-171. doi: 10.1016/j.foodcont.2013.10.025

Siemer, C., Toepfl, S., and Heinz, V. (2014b). Inactivation of Bacillus subtilis spores by pulsed electric fields (PEF) in combination with thermal energy II. Modeling thermal inactivation of B. subtilis spores during PEF processing in combination with thermal energy. Food Control 39, 244-250. doi: 10.1016/j.foodcont.2013.09.067

Thiebaud, M., Dumay, E., Picart, L., Guiraud, J. P., and Cheftel, J. C. (2003). High-pressure homogenisation of raw bovine milk. Effects on fat globule size distribution and microbial inactivation. Int. Dairy J. 13, 427-439. doi: 10.1016/S0958-6946(03)00051-7

Toepfl, S., Siemer, C., Saldaña-Navarro, G., and Heinz, V. (2014). "Chapter 6 Overview of pulsed electric fields processing for food," in Emerging Technologies 
for Food Processing, 2nd Edn., ed D.-W. Sun (San Diego, CA: Academic Press), 93-114.

Vachon, J. F., Kheadr, E. E., Giasson, J., Paquin, P., and Fliss, I. (2002). Inactivation of foodborne pathogens in milk using dynamic high pressure. J. Food Prot. 65, $345-352$.

Valencia-Flores, D. C., Hernández-Herrero, M., Guamis, B., and Ferragut, V. (2013). Comparing the effects of ultra-high-pressure homogenization and conventional thermal treatments on the microbiological, physical, and chemical quality of almond beverages. J. Food Sci. 78, E199-E205. doi: $10.1111 / 1750-3841.12029$

Van Boekel, M. A. J. S. (2002). On the use of the Weibull model to describe thermal inactivation of microbial vegetative cells. Int. J. Food Microbiol. 74, 139-159. doi: 10.1016/S0168-1605(01)00742-5

Van Boekel, M. A. J. S. (2010). Kinetic Modeling of Reactions in Foods. Boca Raton, FL: CRC Press

Wuytack, E. Y., Diels, A. M. J., and Michiels, C. W. (2002). Bacterial inactivation by high-pressure homogenisation and high hydrostatic pressure. Int. J. Food Microbiol. 77, 205-212. doi: 10.1016/S0168-1605(02)0 0054-5

Zamora, A., and Guamis, B. (2015). Opportunities for ultra-high-pressure homogenisation (UHPH) for the food industry. Food Eng. Rev. 7, 130-142. doi: 10.1007/s12393-014-9097-4

Conflict of Interest Statement: The authors declare that the research was conducted in the absence of any commercial or financial relationships that could be construed as a potential conflict of interest.

Copyright $\odot 2015$ Dong, Georget, Aganovic, Heinz and Mathys. This is an openaccess article distributed under the terms of the Creative Commons Attribution License (CC BY). The use, distribution or reproduction in other forums is permitted, provided the original author(s) or licensor are credited and that the original publication in this journal is cited, in accordance with accepted academic practice. No use, distribution or reproduction is permitted which does not comply with these terms. 